\title{
Fibroangioma nasofaríngeo juvenil. Experiencia de 12 años en el Hospital Clínico de la Universidad de Chile
}

\author{
Juvenile nasopharyngeal angiofibroma. 12 years experience \\ at the University of Chile Clinical Hospital
}

Katherine Walker $\mathrm{J}^{1}$, Daniel Muñoz S², Claudio Gaete F³ , Carlos Celedón $\mathrm{L}^{1}$.

\section{RESUMEN}

Introducción: El fibroangioma juvenil es un tumor vascular que afecta casi exclusivamente la nasofaringe de adolescentes de sexo masculino. Su manejo es complejo dada su naturaleza vascular y sus frecuentes recurrencias.

Objetivo: Mostrar la experiencia de 12 años en fibroangioma juvenil

Material y método: Estudio descriptivo retrospectivo de los pacientes que ingresaron con diagnóstico de fibroangioma nasofaríngeo juvenil al Servicio de Otorrinolaringología del Hospital Clínico de la Universidad de Chile entre los años 1996 y 2008, caracterizando al grupo de estudio en cuanto a clínica, diagnóstico, manejo terapéutico, complicaciones y recurrencias.

Resultados: Se obtuvo un total de 22 pacientes, todos de sexo masculino, con un promedio de edad de 17,7 años. El síntoma de presentación más frecuente fue la epistaxis a repetición presente en el $81,8 \%$ de los casos. Todos los pacientes se estudiaron con tomografía computarizada y recibieron embolización arterial preoperatoria. La mayoría de los tumores fueron de tipo II B y II C (68,1\%) según Radkowski y la técnica quirúrgica más empleada fue la endoscópica (54,5\%). Hubo sólo una complicación mayor, pero derivada del procedimiento de embolización y no de la cirugía propiamente tal. Se encontró $22,7 \%$ de recurrencias ( $n=5$, todas endoscópicas), con un tiempo libre de enfermedad de 8,2 meses promedio.

Discusión y conclusión: Nuestros resultados concuerdan con la gran mayoría de las series publicadas en la literatura. La decisión terapéutica se basa en la determinación del tamaño tumoral y su extensión, prefiriendo inicialmente un abordaje endoscópico por su carácter poco invasivo y por presentar menor sangrado, tiempo quirúrgico y estadía hospitalaria; siempre que el estadio tumoral lo permita. La embolización arterial, independiente de la vía de abordaje, debe ser de rutina.

Palabras clave: Fibroangioma, embolización arterial, epistaxis, rinofaringe.

\section{ABSTRACT}

Introduction: Juvenile nasopharyngeal angiofibroma is a vascular tumor that occur almost exclusively in the nasopharynx of adolescent males. Its management is complex because of its vascular nature and frequent recurrence.

Aim: To review the experience of 12 years in juvenile nasopharyngeal angiofibroma.

\footnotetext{
Médico Otorrinolaringólogo. Hospital Clínico Universidad de Chile.

Médico Cirujano. Programa Magíster en Bioestadística, Universidad de Chile.

Médico Residente. Servicio de Otorrinolaringología, Hospital Clínico Universidad de Chile.
} 
Material and method: Retrospective study of patients admitted with diagnosis of juvenile nasopharyngeal angiofibroma at the Otorhinolaryngology Department, University of Chile Clinical Hospital, between 1996 and 2008, characterizing the study group in terms of clinical features, diagnosis, treatment, complications and recurrences.

Results: We obtained a total of 22 patients, all male, with an average age of 17.7 years. The most common presenting symptom was recurrent epistaxis, present in up to $81.8 \%$ of cases. All patients were studied with computed tomography and received preoperative arterial embolization. Most tumors were type II B and C (68.1\%) and the endoscopic surgical technique was predominant. There was only one major complication, but derived from the embolization procedure and not the surgery itself. There was a $22.7 \%$ recurrence, with disease-free time of 8.2 months on average.

Discussion and conclusion: Our results agree with most of the series published in the literature. The therapeutic decision is based on the determination of tumor size and extension, preferring an initial endoscopic approach, for its minimally invasive nature and because they present less bleeding, surgical time and hospital stay, if the tumor stage allows. Arterial embolization, regardless of the surgical approach, should be routine.

Key words: Angiofibroma, arterial embolization, epistaxis, rhinopharynx.

\section{INTRODUCCIÓN}

El fibroangioma nasofaríngeo juvenil (FNJ) es una neoplasia vascular benigna, infrecuente, que da cuenta de menos del 0,5\% de todos los tumores de cabeza y cuello ${ }^{1}$. Ocurre casi exclusivamente en la nasofaringe de adolescentes de sexo masculino $0^{2,3} y$ su sitio de origen es controversial ${ }^{4}$. Los FNJ tiene un crecimiento lento y se expanden en un comienzo dentro del la cavidad nasal y nasofaringe y luego en la fosa pterigomaxilar. Con el tiempo, eventualmente pueden erosionar el hueso e invadir la fosa infratemporal, órbita y fosa craneal media. El aporte sanguíneo habitualmente proviene de la arteria maxilar interna, pero también puede provenir de la arteria carótida interna 0 de la arteria faríngea ascendente ${ }^{5,6}$.

Los FNJ clásicamente se presentan con obstrucción nasal unilateral, epistaxis recurrente 0 tumoración nasal ${ }^{7,8}$. Se describen otras manifestaciones relacionadas con la extensión tumoral, tales como hipoacusia de conducción, dacriocistitis, deformidad de paladar, parálisis facial, proptosis y hemorragia masiva9,10.

Durante la evaluación inicial puede usarse la tomografía computarizada y la resonancia nuclear magnética para determinar la extensión del tumor. La tomografía computarizada es excelente para la evaluación del detalle óseo y extensión tumoral sobre todo con medio de contraste. Además, el abombamiento característico de la pared posterior del maxilar debido a la presencia de la masa en el espacio pterigomaxilar, conocido como signo de Holman-Miller, es un hallazgo típico de este examen $^{11}$. La resonancia nuclear magnética permite evaluar los tejidos blandos y diferenciar el tumor de inflamación de mucosa, fluidos sinusales y estructuras como el seno cavernos0 $0^{12,13}$. Según Nicolai y cols, la resonancia magnética fue eficaz en detectar la erosión del proceso pterigoideo, extensión hacia la fosa pterigomaxilar e infratemporal, cambios que fueron luego comprobados durante la cirugía ${ }^{22}$.

Existe una variedad de clasificaciones de estos tumores. La clasificación de Radkowski, desarrollada en 1996 es la más utilizada en la literatura de los últimos años. Existen además otras clasificaciones como la de Fisch, Andrews y Sessions. Todas ellas se basan en la extensión e invasión de estructuras por el tumor, ${ }^{6,14}$ (Tabla 1).

La cirugía es el tratamiento de primera elección ${ }^{15}$. La embolización arterial supraselectiva preoperatoria de ramas de la carótida externa ha disminuido significativamente las pérdidas sanguíneas y ha facilitado la resección de tumores de gran tamaño. Es en general exitosa y sus complicaciones son la mayoría de las veces menores $^{16,17}$.

Los avances en cirugía endoscópica nasal y la habilidad de embolizar los tumores vasculares han hecho que esta técnica sea cada vez más usada en la actualidad ${ }^{18-21}$. Alternativamente al tratamiento 
Tabla 1. Sistemas de clasificación de fibroangiomas según Radkowski, Sessions, Fisch y Andrews

\begin{tabular}{|c|c|c|c|}
\hline Radkowski & Sessions & Fisch & Andrews \\
\hline $\begin{array}{l}\text { IA. Tumor limitado cavidad } \\
\text { nasal y/o nasofaringe }\end{array}$ & $\begin{array}{l}\text { IA. Limitado a fosa nasal } \\
\text { posterior y/o arco coanal }\end{array}$ & $\begin{array}{l}\text { I. Tumor limitado a naso- } \\
\text { faringe y cavidad nasal } \\
\text { sin destrucción ósea }\end{array}$ & I. Limitado a cavidad nasal \\
\hline $\begin{array}{l}\text { IB. Igual que IA pero con } \\
\text { compromiso de al menos } \\
\text { una cavidad paranasal }\end{array}$ & $\begin{array}{l}\text { IB. Engloba fosa nasal } \\
\text { posterior y/o arco coanal } \\
\text { con afección de al menos } \\
\text { un seno paranasal }\end{array}$ & $\begin{array}{l}\text { II. Tumor que invade la } \\
\text { fosa pterigomaxilar y los } \\
\text { senos maxilar, etmoides } \\
\text { y esfenoides con } \\
\text { destrucción ósea }\end{array}$ & $\begin{array}{l}\text { II. Invasión de fosa } \\
\text { pterigopalatina, seno } \\
\text { maxilar, etmoides } 0 \\
\text { esfenoides }\end{array}$ \\
\hline $\begin{array}{l}\text { ॥A. Extensión mínima a } \\
\text { través del agujero } \\
\text { esfenopalatino y compromiso } \\
\text { lateral mínimo hacia la } \\
\text { fosa pterigomaxilar }\end{array}$ & $\begin{array}{l}\text { IIA. Extensión lateral } \\
\text { mínima a la fosa } \\
\text { pterigomaxilar }\end{array}$ & $\begin{array}{l}\text { III. Invade la fosa } \\
\text { infratemporal, la órbita } \\
\text { y la región paraselar } \\
\text { pero permanece lateral } \\
\text { al seno cavernoso }\end{array}$ & $\begin{array}{l}\text { IIIA. Fosa infratemporal, } \\
\text { fosa orbitaria sin afección } \\
\text { intracraneal }\end{array}$ \\
\hline $\begin{array}{l}\text { IIB. Ocupación completa de } \\
\text { la fosa pterigomaxilar, } \\
\text { desplazando hacia anterior } \\
\text { la pared posterior del antro } \\
\text { maxilar. Extensión superior } \\
\text { con o sin erosión de los } \\
\text { huesos orbitarios }\end{array}$ & $\begin{array}{l}\text { IIB. Ocupación plena de } \\
\text { fosa pterigomaxilar con } \\
\text { o sin erosión de hueso } \\
\text { orbitario }\end{array}$ & $\begin{array}{l}\text { IV. Tumor con invasión } \\
\text { masiva del seno } \\
\text { cavernoso, de la región } \\
\text { del quiasma óptico } \\
\text { y la celda hipofisiaria }\end{array}$ & $\begin{array}{l}\text { IIIB. Estadio IIIA con } \\
\text { afección intracraneal } \\
\text { extradural (paraselar) }\end{array}$ \\
\hline $\begin{array}{l}\text { IIC. Extensión hacia la fosa } \\
\text { infratemporal o hacia } \\
\text { posterior hasta los procesos } \\
\text { pterigoídeos }\end{array}$ & $\begin{array}{l}\text { IIIA. Erosión de base de } \\
\text { cráneo (p. ej., fosa craneal } \\
\text { media, base pterigoídea), } \\
\text { extensión intracraneal mínima }\end{array}$ & - & $\begin{array}{l}\text { IVA. Intradural sin } \\
\text { afección de seno } \\
\text { cavernoso, pituitaria o } \\
\text { quiasma }\end{array}$ \\
\hline $\begin{array}{l}\text { IIIA. Erosión de base de } \\
\text { cráneo con mínima } \\
\text { extensión intracraneana }\end{array}$ & $\begin{array}{l}\text { IIIB. Extensión intracraneal } \\
\text { extensa con o sin invasión } \\
\text { del seno cavernoso }\end{array}$ & - & $\begin{array}{l}\text { IVB. Afección de seno } \\
\text { cavernoso, pituitaria } \\
\text { o quiasma }\end{array}$ \\
\hline $\begin{array}{l}\text { IIIB. Erosión de base de } \\
\text { cráneo. Compromiso } \\
\text { intracraneal extenso con o sin } \\
\text { extensión al seno cavernoso }\end{array}$ & - & - & - \\
\hline
\end{tabular}

quirúrgico, se han desarrollado la terapia hormonal, la radioterapia y la quimioterapia en el manejo de los FNJ23-26.

Con el fin de averiguar cuál es la realidad local respecto al diagnóstico, tratamiento, recidivas y compararla con publicaciones internacionales, se realizó un análisis retrospectivo de los pacientes con diagnóstico de FNJ ingresados al Servicio de
Otorrinolaringología del Hospital Clínico de la Universidad de Chile.

\section{MATERIAL Y MÉTODO}

Estudio descriptivo retrospectivo. Se revisaron las historias clínicas de los pacientes ingresados al 
Servicio de Otorrinolaringología del Hospital Clínico de la Universidad de Chile entre los años 1996 y 2008, con diagnóstico de FNJ. Se obtuvieron datos clínico-epidemiológicos, métodos diagnósticos, características tumorales, modalidades de tratamiento, recidivas y presencia de complicaciones. Para el análisis de los datos se formaron dos grupos: el primero, compuesto por aquellos pacientes intervenidos por vía endoscópica y el segundo, compuesto por aquellos pacientes intervenidos por vía abierta. Los datos se tabularon y analizaron mediante el software estadístico Stata 10.1, utilizando la prueba no paramétrica $U$ de Mann-Whitney para la comparación de medias. Se consideró como significativo un valor $p<0,05$.

\section{RESULTADOS}

Durante el periodo de tiempo revisado, se obtuvo un total de 22 pacientes intervenidos quirúrgicamente en nuestro servicio, todos de sexo masculino, con un promedio de edad de 17,7 años y sin antecedentes mórbidos de importancia. El síntoma de presentación más frecuente fue la epistaxis a repetición presente en el $59 \%$ de los casos, seguida de la obstrucción nasal y, en algunos casos, la combinación de ambas (Figura 1). Los pacientes atendidos presentaban un promedio de 7,7 meses de evolución de sus síntomas al momento de la consulta.

Todos fueron estudiados nasofibroscópicamente y con tomografía computarizada de cavidades paranasales con medio de contraste para estudio de extensión tumoral. En el 18,1\% (4/22) se requirió el uso de resonancia magnética para detallar la extensión hacia estructuras más profundas. La totalidad de los pacientes fueron sometidos a angiografía y embolización arterial preoperatoria. Según esto, se obtuvo que el aporte sanguíneo de estos tumores fue dado principalmente por las arterias maxilar interna $(59 \%)$, esfenopalatina $(22,7 \%)$ y faríngea ascendente $(18,1 \%)$ (Figura 2).

De acuerdo al sistema de clasificación de estos tumores, se encontró que el 13,5\% (3/22) correspondió a estadio I, 77,1\% (17/22) estadio II y $9 \%$ (2/22) estadio III. El desglose según la clasificación de Radkowski se detalla en la Tabla 2.

La técnica quirúrgica más empleada fue la endoscópica transnasal en el 54,5\% (12/22). En el resto de los casos se usó la técnica abierta con abordaje por degloving mediofacial. Hubo sólo una complicación mayor (amaurosis de ojo derecho), pero derivada del procedimiento de embolización y no de la cirugía propiamente tal. En todos los casos el análisis histopatológico comprobó el diagnóstico clínico de fibroangioma juvenil. El taponamiento nasal fue retirado en promedio 3,2 días poscirugía.

Dentro de las características tumorales destaca que para aquellos tumores resecados endoscópicamente, el tamaño promedio (medido antes de la embolización) fue de $4,6 \mathrm{~cm}$, mientras que aquellos tumores resecados por vía abierta el tamaño promedio fue de $5,5 \mathrm{~cm}$. Asimismo, el tiempo de cirugía para la vía endoscópica fue 3,3 horas

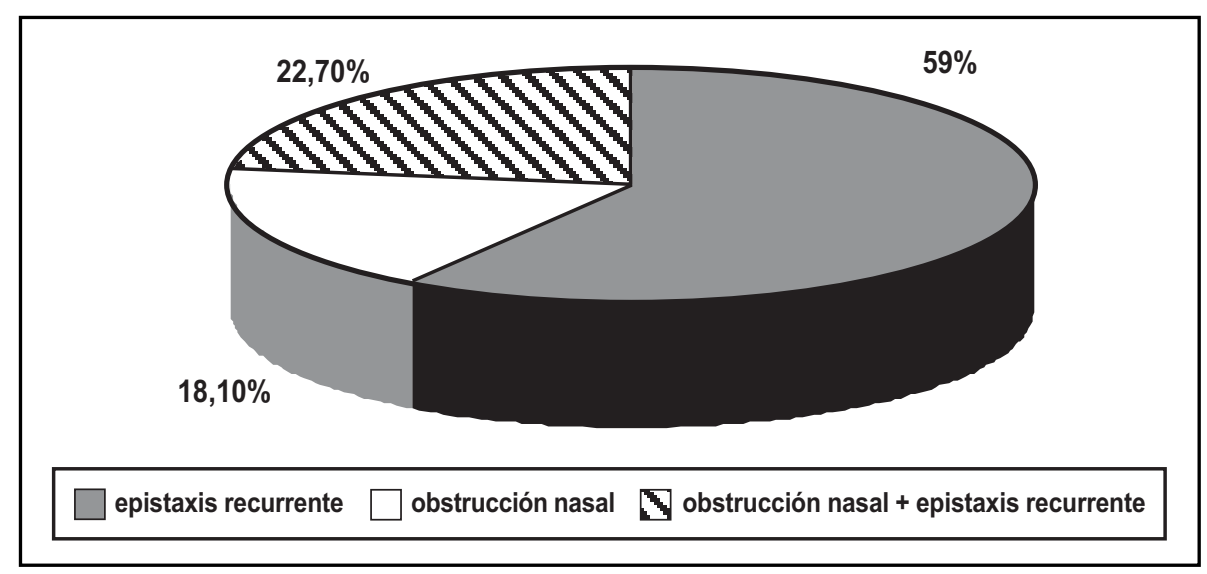

Figura 1. Motivos de consulta de los pacientes con fibroangioma, HCUCH 1996-2008. 


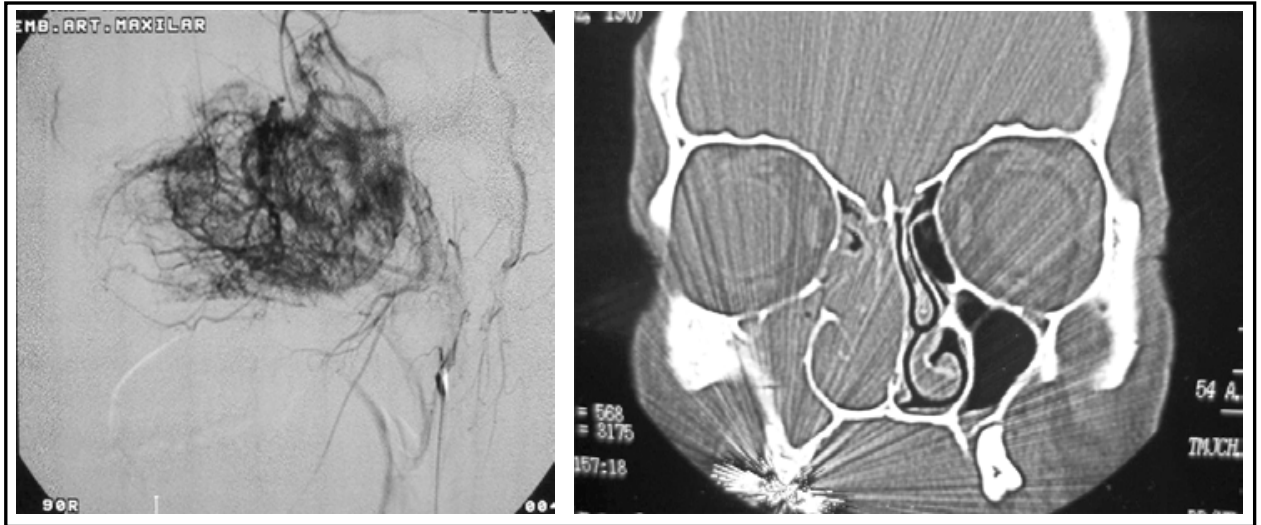

Figura 2. A la izquierda, se observa la angiografía y embolización arterial de un fibroangioma nasofaríngeo. En este caso se emboliza un paquete vascular proveniente de la arteria maxilar. A la derecha, la tomografía computarizada del mismo caso, donde se aprecia el compromiso tumoral de la fosa nasal derecha y su extensión hacia el seno maxilar.

Tabla 2. Distribución de los pacientes por estadio tumoral, según la clasificación de Radkowski

\begin{tabular}{|lrr|}
\hline Estadio & N $^{\circ}$ & $\%$ \\
\hline I A & 1 & 4,5 \\
I B & 2 & 9 \\
II A & 2 & 9 \\
II B & 10 & 45,4 \\
II C & 5 & 22,7 \\
III A & 2 & 9 \\
III B & 0 & 0 \\
\hline Total & 22 & 100 \\
\hline
\end{tabular}

promedio, mientras que para la vía abierta se tardó en promedio 4 horas. Por otro lado, aquellos pacientes intervenidos endoscópicamente estuvieron en promedio 4,3 días hospitalizados, mientras que aquellos operados por vía abierta permanecieron en promedio 8,9 días en el hospital. Esta última variable fue la única que mostró una diferencia estadísticamente significativa ( $p=0,04)$ (Tabla 3).

En un caso fue necesario el uso de radioterapia, ya que se trató de un paciente con recidiva tumoral, que en el posoperatorio inmediato presentó evidencia de resección incompleta de la lesión. La respuesta a radioterapia fue óptima, apreciándose la involución completa de la masa tumoral residual postratamiento. En nuestra serie evidenciamos in- vasión intracraneana o erosión de base de cráneo en el $9 \%$ de los pacientes.

Con respecto a la recidiva tumoral, encontramos su presencia en el 22,7\% (5/22) de los casos, siendo 4 de ellos la primera recidiva y un caso de una tercera recidiva, en estadios IIIA ( $n$ $=2)$ y IIC $(n=3)$. Todos ellos fueron resecados por vía endoscópica como primer tratamiento, lo que entrega $41,6 \%$ de tasa de recidiva endoscópica. Se estimó para estos casos, un tiempo libre de enfermedad de 8,2 meses promedio.

\section{DISCUSIÓN}

La muestra estudiada reflejó similitudes, tanto en el tamaño muestral como en características clínicoepidemiológicas, con respecto a algunas series reportadas ${ }^{7,12,22}$. La edad de presentación y la predominancia (en nuestro caso absoluta) por el sexo masculino, se correlaciona con la descripción clásica de los fibroangiomas juveniles nasofaríngeos ${ }^{1,2}$.

En nuestra serie los síntomas y la latencia de consulta concuerdan con varios reportes similares en cuanto a los síntomas principales y el tiempo de evolución de la sintomatología ${ }^{22}$. Se dice habitualmente, que el curso natural de la enfermedad es variable pero con cierta tendencia al diagnóstico tardío, pues los síntomas más frecuentes están dados fundamentalmente por tumores en estadios avanzados ${ }^{8}$. 
Tabla 3. Características tumorales y resultados, según modalidad de tratamiento

\begin{tabular}{|lccc|}
\hline & $\begin{array}{c}\text { Vía endoscópica } \\
(\mathrm{n}=12)\end{array}$ & $\begin{array}{c}\text { Vía abierta } \\
(\mathrm{n}=10)\end{array}$ & $p$-value \\
\hline Tamaño tumoral (cm)* & $4,6 \pm 2$ & $5,5 \pm 1,5$ & 0,2 \\
Tiempo de cirugía (hrs) & $3,3 \pm 2$ & $4 \pm 1,2$ & 0,1 \\
Pérdidas de sangre (ml) & $450 \pm 60$ & $650 \pm 100$ & 0,1 \\
Estadía hospitalaria (días) & $4,3 \pm 2$ & $8,9 \pm 2,5$ & 0,04 \\
Retiro taponamiento (días) & $2,8 \pm 1$ & $3,6 \pm 1,2$ & 0,2 \\
Recurrencia & $41,6 \%$ & $0 \%$ & - \\
\hline
\end{tabular}

*Tamaño estimado antes de la embolización arterial.

A pesar de su naturaleza benigna, los fibroangiomas poseen un patrón de crecimiento agresivo. La invasión intracraneal, que puede afectar hasta el $36 \%$ de los pacientes según algunos autores $^{27}$, ocurre habitualmente de forma extradural a nivel de fosa craneal media y raramente hacia fosa anterior ${ }^{28}$.

La modalidad de tratamiento adoptada depende fundamentalmente del tamaño y extensión tumoral, junto con las características del centro de salud y las habilidades del equipo quirúrgico. El objetivo de la cirugía es la resección completa, tratando de minimizar la morbilidad y las pérdidas de sangre, de acuerdo con los principios de la resección en block de la cirugía oncológica ${ }^{29}$. En nuestra institución se ha usado principalmente la técnica endoscópica y la vía abierta con técnica degloving mediofacial, en caso de localizaciones y extensiones complejas.

La cirugía endoscópica ofrece la ventaja de poseer una visión en varios ángulos del tumor y estructuras adyacentes, lo que es fundamental para lograr un buen control del sangrado intraoperatorio. Además, esta técnica no requiere incisiones de piel 0 mucosas, ni elevación de tejidos blandos de la pared anterior del maxilar, remoción de hueso, osteotomías faciales, entre otras ${ }^{30,31}$.

Varios estudios han usado variables como el tamaño tumoral, el tiempo de cirugía, sangrado intraoperatorio, el tiempo de estadía hospitalaria y el retiro del taponamiento nasal, entre otros, como parámetros para evaluar la respuesta a tratamiento y con el fin de comparar las distintas modalidades. Según esto, la modalidad endoscópica ha mostra- do diferencias favorables en las distintas variables estudiadas, sobre todo en tumores en estadios tempranos ${ }^{12,21,22}$.

En nuestro servicio es de rutina la indicación de embolización arterial supraselectiva preoperatoria en fibroangiomas primarios y en recidivas, ya que es indiscutible su beneficio para estos tumores vascularizados pues disminuye el tamaño tumoral, la hemorragia intraoperatoria, facilita la resección completa y, por tanto, acorta el tiempo quirúrgi$\mathrm{C} 0^{13,34}$, constituyendo así un punto clave en el manejo de estos pacientes.

La recurrencia 0 recidiva tumoral se define como la presencia de tumor clínica 0 radiológicamente en nasofaringe, fosa nasal 0 estructuras vecinas y con evidencia de crecimiento derivada de la aparición de síntomas luego de 6 meses del tratamiento inicial ${ }^{32}$. En la literatura se reportan tasas de recurrencias variables, de entre $13 \%$ y $50 \%$. Esta amplia variabilidad se relaciona principalmente a la distribución de pacientes en las distintas series publicadas, en términos de la estadificación y los criterios (clínicos o radiológicos) usados para establecer la presencia de una lesión residual. Según Carrillo y $\operatorname{cols}^{10}$, la tasa de recurrencias dependería del tamaño y extensión tumoral, la embolización previa, el uso de radioterapia y las características de los márgenes quirúrgicos.

Resulta de importancia, por lo tanto, un seguimiento estricto de la evolución de los pacientes intervenidos, mediante evaluación clínica y el uso de imágenes. Según Herman y $\operatorname{cols}^{33}$, la tasa de identificación de recidivas mediante tomografía 
computarizada fue de $7 \%$, lo que aumenta a $39,5 \%$ en pacientes con y sin compromiso de base de cráneo.

En nuestro hospital existe una experiencia de 20 años, presentada por Celedón y cols ${ }^{35}$ en 1995. En esta serie de 34 pacientes diagnosticados, el $84,8 \%$ recibió una modalidad terapéutica única (cirugía, escleroterapia o radioterapia) mientras el resto recibió la combinación de estas técnicas, siendo la cirugía el denominador común en todos Ios casos. El abordaje quirúrgico varió dependiendo de la localización específica del tumor, extensión y año en el período de estudio. Las complicaciones fueron mínimas, destacando la hemorragia posoperatoria, necrosis del paladar y los problemas estético-funcionales.

\section{CONCLUSIONES}

EI FNJ es un tumor vascular de rinofaringe, infrecuente en la población general, que se presenta casi exclusivamente en varones adolescentes. La remoción quirúrgica corresponde al tratamiento de elección, con tendencia en los últimos años al uso preferente de técnicas endoscópicas y al uso universal de la embolización arterial preoperatoria. Dado el comportamiento tumoral es necesario un frecuente control posoperatorio, que debe incluir endoscopía nasal y/o tomografías computarizadas con contraste para la pesquisa de recidivas. En el Hospital Clínico de la Universidad de Chile, el manejo de esta patología se engloba en las tendencias internacionales de diagnóstico, tratamiento y seguimiento de estos pacientes.

\section{BIBLIOGRAFÍA}

1. Bremer JW, Neel hB III, de Santo LW. Angiofibroma: Treatment trends in 150 patients during 40 years. Laryngoscope 1986; 96: 1321-9.

2. Kau R, tanna M, Misra S, Contreras al. Nasopharyngeal angiofibroma. Ear Nose Throat J 2008; 87(5): 256.

3. Montero X, Platzer L, Castillo M. Actualizaciones en tumores de rinofaringe. Rev Otorrinolaringol Cir Cabeza Cuello 2005; 65: 221-32.
4. Hosseini SM, Borghel P, Borghel SH, Ashtiani MT, ShIRKHODA A. Angiofibroma: an outcome review of conventional surgical approaches. Eur Arch Otorhinolaryngol 2005; 262(10): 807-12.

5. Sennes LU, Butugan 0, Sánchez TG, Bento RF, Tsuj $\mathrm{DH}$. Juvenile nasopharyngeal angiofibroma: the routes of invasion. Rhinology 2003; 41(4): 235-40.

6. Hauptman $G$, Ulualp $S$, Quinn $F$. Juvenile Nasopharyngeal Angiofibroma. Grand Rounds Presentation, UTMB, Dept. of Otolaryngology. 3 de Enero, 2007. http://www.utmb.edu/otoref/ grnds/GrndsIndex.html [Consulta, Octubre de 2009].

7. Yadav SP, Singh I, Chanda R, Sachdeva OP. Nasopharyngeal angiofibroma. J Otolaryngol 2002; 31(6): 346-50.

8. Paris J, Guelfucci B, Moulin G, Zanaret M, Triglia JM. Diagnosis and treatment of juvenile nasopharyngeal angiofibroma. Eur Arch Otorhinolaryngol 2001; 258(3): 120-4.

9. Rao BN, Shewalkar BK. Clinical profile and multimodality approach in the management of juvenile nasopharyngeal angiofibroma. Indian J Cancer 2000; 37(4): 133-9.

10. Carrillo Jf, Maldonado F, Albores 0, RamírezOrtega MC, OÑate-Ocaña LF. Juvenile nasopharyngeal angiofibroma: Clinical factors associated with recurrence, and proposal of a staging system. J Surg Oncol 2008; 98(2): 75-80.

11. Kania Re, Sauvaget E, Guichard JP, Chapot R, Huy PT, Herman P. Early postoperative CT scanning for juvenile nasopharyngeal angiofibroma: detection of residual disease. AJNR Am J Neuroradiol 2005; 26(1): 82-8.

12. Scholtz AW, Appenroth E, Kammen-Jolly K, Scholtz LU, THumfart WF. Juvenile nasopharyngeal angiofibroma: management and therapy. Laryngoscope 2001; 111(4 Pt 1): 681-7.

13. Gupta AK, PuRKayastha S, Bodhey NK, Kapilamoorthy TR, Kesavadas C. Preoperative embolization of hypervascular head and neck tumours. Australs Radiol 2007; 51(5): 446-52.

14. Radkowski D, McGill T, Healy GB, Ohlms L, Jones DT. Angiofibroma. Changes in staging and treatment. Arch Otolaryngol Head Neck Surg 1996; 122(2): 122-9.

15. ENEPEKIDES DJ. Recent advances in the treatment of juvenile angiofibroma. Curr Opin Otolaryngol Head Neck Surg 2004 Dec; 12(6):495-499. 
16. Hemingway AP, Aluison DJ. Complications of Embolization: Analysis of 410 Procedures. Radiology 1988; 166: 669-72.

17. Cansiz H, Guvenc MG, Sekecioglu N. Surgical approaches to juvenile nasopharyngeal angiofibroma. J Craniomaxillofac Surg 2006; 34(1): 3-8.

18. Eloy P, Watelet JB, Hatert AS, de Wispelaere J, BERTRAND B. Endonasal endoscopic resection of juvenile nasopharyngeal angiofibroma. Rhinology 2007; 45(1): 24-30.

19. Douglas R, Wormald PJ. Endoscopic surgery for juvenile nasopharyngeal angiofibroma: where are the limits? Curr Opin Otolaryngol Head Neck Surg 2006; 14(1): 1-5.

20. Pryor SG, Moore EJ, Kasperbauer JL. Endoscopic versus traditional approaches for excision of juvenile nasopharyngeal angiofibroma. Laryngoscope 2005; 115(7): 1201-7.

21. Danesi G, Panciera dT, Harvey RJ, Agostinis C. Juvenile nasopharyngeal angiofibroma: evaluation and surgical management of advanced disease. Otolaryngol Head Neck Surg 2008; 138(5): 581-6.

22. Nicolal P, Berlucchi M, Tomenzoli D, Cappiello J, Trimarchi M, Maroldi R, Battaglia G, Antonelli AR. Endoscopic surgery for juvenile angiofibroma: when and how. Laryngoscope 2003; 113(5): 775-82.

23. Lee JT, Chen P, Safa A, Juillard G, Calcaterra TC. The role of radiation in the treatment of advanced juvenile angiofibroma. Laryngoscope 2002; 112(7 Pt 1): 1213-20.

24. Schick B, Kahle $G$, Hassler R, Draf W. Chemotherapy of juvenile angiofibroma-an alternative? HNO 1996; 44(3): 148-52.

25. Gates GA, Rice DH, Koopmann CF JR. Flutamideinduced regression of angiofibroma. Laryngoscope 1992; 102: 641-4.

26. Labra A, Chavolla-Magana R, López-Ugalde A, Alanis-Calderón J, Huerta-Delgado A. Flutamide as a preoperative treatment in juvenile angiofibroma $(\mathrm{JA})$ with intracranial invasion: report of 7 cases. Otolaryngol Head Neck Surg 2004; 130(4): 466-9.

27. Close LG, Schaefer SD, Mickey Be, Manning SC. Surgical management of nasopharyngeal angiofibromas involving the cavernous sinus. Arch Otolaryngol Head Neck Surg 1989; 115: 1091-5.

28. HaRMA RA. Nasopharyngeal angiofibroma: a clinical and histopathological study. Acta Otolaryngol (Suppl) 1958; 146: 1-76.

29. Marshall AH, Bradley PJ. Management dilemmas in the treatment and follow-up of advanced juvenile nasopharyngeal angiofibroma. ORL $J$ Otorhinolaryngol Relat Spec 2006; 68(5): 273-8.

30. Carrau RL, Snyderman CH, Kassam AB, Jungreis CA. Endoscopic and endoscopic-assisted surgery for juvenile angiofibroma. Laryngoscope 2001; 111(3): 483-7.

31. KHALIFA MA. Endonasal endoscopic surgery for nasopharyngeal angiofibroma. Otolaryngol Head Neck Surg 2001; 124(3): 336-7

32. Howard DJ, LLoYd G, Lund V. Recurrence and its avoidance in juvenile angiofibroma. Laryngoscope 2001; 111(9): 1509-11.

33. Herman P, Lot G, Chapot R, Salvan D, Huy PT. Long-term follow-up of juvenile nasopharyngeal angiofibromas: analysis of recurrences. Laryngoscope 1999;109(1): 140-7.

34. Aedo C, Naser A, Cabello P, Muñoz D, Palavicino $P$, PriEto J. Embolización arterial supraselectiva en otorrinolaringología: Indicaciones y complicaciones en 12 años. Rev Otorrinolaringol Cir Cabeza Cuello 2008; 68: 149-56.

35. Celedón C, Pruzzo E, Rico B, Sepúlveda J, Navarrete C. Fibroangioma de rinofaringe: experiencia de 20 años. Rev Otorrinolaringol Cir Cabeza Cuello 1995; 55(2/3): 65-71. 